\title{
TRYPTAMINE DERIVATIVES OF SESQUITERPENE LACTONES PREVENT THE B-AMYLOID FORMATION AND AGGREGATION
}

\author{
Yu.R. Aleksandrova' ${ }^{1}$, A.V. Semakov', S.G. Klochkov', E.Yu. Yandulova', \\ M.E. Neganova ${ }^{1}$
}

${ }^{1}$ IPAC RAS, 142432, Russia, Chernogolovka, Severniy pr., 1.

DOI: 10.19163/MedChemRussia2021-2021-329

E-mail: yulia.aleks.97@mail.ru

The existing data set of histopathological, genetic and biomarker analyses confirms the fact that the formation, aggregation and deposition of pathological forms of $\beta$-amyloid occur long before the onset of dementia symptoms. At the same time, the key role in triggering the amyloidogenic pathway of the amyloid precursor protein cleavage, which results in the formation of insoluble and aggregation-prone $A \beta$ fragments, is the enzyme $\beta$-secretase 1 (BACE1). Therefore, a promising strategy in the development of therapeutic agents for the treatment of Alzheimer's disease is to create drugs targeting Aß.

As part of the synthetic work, hybrid molecules based on sesquiterpene lactones and tryptamine analogues were obtained, and their potential neuroprotective properties were studied in a series of experiments in vitro and in vivo.

An in vitro study of the neuroprotective potential of new aminoderivatives of sesquiterpene lactones revealed a leader compound - conjugate of alloalantholactone with 5-methoxytryptamine, which was shown to have the ability to suppress the BACE1 enzyme activity, as well as to exhibit antiaggregational properties against $A \beta_{1-42}$, inhibiting this process by more than $50 \%$. The absence of a pronounced toxic effect on a number of cell cultures made it possible to study its neuroprotective properties in in vivo experiments on 5xFAD animals used to model Alzheimer's disease. It was found that this compound has the ability to restore the lost cognitive functions in transgenic mice and improve spatial learning and memory in the «new object recognition» and «Morris water maze» tests.

Thus, the results obtained confirm the prospects of a synthetic approach to the creation of effective neuroprotective compounds, the target of which is the pathological $A \beta$ formation and aggregation, and the selected leader compound can be considered as a basis for the creation of drugs for the Alzheimer's disease treatment. 